\title{
The laboratory investigation of the innovative sensor for torsional effects in engineering structures' monitoring
}

\author{
A. KURZYCH*1, J.K. KOWALSKI ${ }^{2}$ B. SAKOWICZ ${ }^{3}$, Z. KRAJEWSKI ${ }^{1}$ and L.R. JAROSZEWICZ ${ }^{1}$ \\ ${ }^{1}$ Institute of Applied Physics, Military University of Technology, ul. Kaliskiego 2, 00-908 Warsaw, Poland \\ ${ }^{2}$ m-Soft Ltd., ul. Sotta Sokoła 4-9, 02-790 Warsaw, Poland \\ ${ }^{3}$ Dep. of Microelectronics and Computer Science, Lodz University of Technology, ul. Wólczańska 221/223, \\ 90-924 Łódź, Poland
}

\begin{abstract}
The main objective of this work is to characterize the performance of an interferometric fibre sensor which has been designed in order to register rotational phenomena, both in seismological observatories and engineering constructions. It is based on a well-known Sagnac effect which enables to detect one-axis rotational motions in a direct way and without any reference system. The presented optical fibre sensor - FOSREM allows to measure a component of rotation in a wide range of signal amplitude form $10^{-8} \mathrm{rad} / \mathrm{s}$ to $10 \mathrm{rad} / \mathrm{s}$, as well as frequency from $0 \mathrm{~Hz}$ to the upper frequency from $2.56 \mathrm{~Hz}$ to $328.12 \mathrm{~Hz}$. The laboratory investigation of our system indicated that it keeps theoretical sensitivity equal to $2 \cdot 10^{-8} \mathrm{rad} / \mathrm{s} / \mathrm{Hz} 1 / 2$ and accuracy no less than $3 \cdot 1^{-8}$ to $1.6 \cdot 10^{-6} \mathrm{rad} / \mathrm{s}$ in the above mentioned frequency band. Moreover, system size that equals $0.36 \times 0.36 \times 0.16 \mathrm{~m}$ and opportunity to remotely control the system via Internet by special server make FOSREM a mobile and autonomous device.
\end{abstract}

Keywords: torsional effects, interferometer, sensor, engineering construction, rotational seismology.

\section{Introduction}

From the beginning of this century, a new approach for seismology is observed which is no longer based only on the analysis of observations made with classical seismometers measuring translational motions or their time derivatives. As described in Igel et al. [1] more and more observables like strain rotations GPS-based measurements and other are used to constrain either the Earth's structure or to understand the nature of seismic source and observe ground shaking. For the above reason the analysis of rotational ground motions has recently emerged as a new branch in seismology and earthquake engineering described as rotational seismology and its engineering applications [2]. As wrote Igel et al. [1] it does not seem to be common, seismological knowledge that particularly concerns motions at the Earth's surface, with (almost any) translational ground motion, there is associated rotational ground motion, as well. Moreover, the theoretical investigation denotes that for rocks with: specific microstructures [3], defects [4], without inner structure [5,6] exist in grainy rocks rotational seismic events as rotational phenomena even as rotational seismic waves [7]. The above statement has several implications summarized in four main

*e-mail: anna.kurzych@wat.edu.pl points in [1]. Neglecting of the rotational motion type so far is basically explained by a tremendous difficulty in measuring these motions with high accuracy in a broad frequency band required in seismology. Recent developments in rotational sensors based on mechanical [8], as well as optical $[9,10]$ principles of operation, produce the first direct measurements that could be compared with theoretical expectations [11] or used to predict the responses of structures [12].

Parallel to the above, current achievements in civil engineering connected with application of innovative materials give opportunity to design and construct high, irregular and complex engineering structures where torsional vibrations are caused by their asymmetry where the centre of stiffness differs from the centre of mass [16]. Such structural vibrations were very often neglected because one thinks that their influence is insignificant or because of lack of suitable instruments to investigate them [14] despite estimating large amplitude of rotational motion even up $10 \mathrm{rad} / \mathrm{s}$ [15]. Such effects can cause damages, therefore, the data on rotation about vertical axes can be implemented during design process in order to increase safety. Torsional vibrations in buildings are accompanied with translational movements in components located away from the rotation centre. The torsional response can be measured by two perpendicularly translational sensors but it has limitations resulting from their 
principle of operation [16]. However, the inherent sensor drift and the small offset from zero in the absence of an input signal are the important limitations of this technique. According to the measurement of interstory drifts, it is, in principle, possible to arrange a frame from the floor below to near the ceiling above to set up displacement transducers to measure the difference in displacements [17]. However, again far from the hardware complexity of this approach, it is also vulnerable to building's deformations. Additionally, during earthquakes, where all is in moving, there is a problem with proper a choice of an external reference frame for such measurement [18]. For the above reason, one of the most promised techniques seems to be sensors based on the Sagnac effect [19] which allows to detect rotation rate in civil engineering constructions without any reference point. The first results of such approach by an application of a commercially available fiber optic gyroscope (FOG) to measure rotation of the flexible engineering structure are presented by Schreiber et al. [14] and Zembaty et al. [20].

Presented in this paper fibre sensor - FOSREM (Fibre-Optic System for Rotational Events \& Phenomena Monitoring) is designed as a rotational seismometer for investigating torsional effects in engineering constructions. Such research can be treated as an area of interest of the strong motion seismology [2] according to the expected high amplitude of rotation - even up $10 \mathrm{rad} / \mathrm{s}$. Construction of FOSREM based on authors' experiences regarding construction and investigation [21] of previous systems designed for continuing monitoring of rotational events connected with earthquakes $[22,23]$, mainly AFORS (Autonomous Fibre-Optic Rotational Seismograph) installed in the seismological observatory in Książ, Poland. In comparison to AFORS, by changes in optical, as well as electronical part, FOSREM protects detection rotation rate with a higher amplitude (up to 10 $\mathrm{rad} / \mathrm{s}$ compare to $10^{-6} \mathrm{rad} / \mathrm{s}$ for AFORS); enables to measure a rotational component in a wide frequency band (from $0 \mathrm{~Hz}$ to $328.12 \mathrm{~Hz}$ ), and is more portable devices which makes it work within the temperature range of $0-50^{\circ} \mathrm{C}$. Additionally, changes in data acquisition system previously described for AFORS [24], the FOSREM protects also elimination of signal artefacts observed during more than five years of a continuous field work of the AFORS.

\section{FOSREM construction}

The project and construction of FOSREM [Fig. 1(a)] have been established in the Institute of Applied Physics, Faculty of Advanced Technologies and Chemistry of the Military University of Technology, Warsaw, Poland based on the continuously performing sensor system AFORS, [Fig. 1(b)] whose data including measuring results are presented inter alia in papers [21-24]. Regarding more compactness, as well as other detection range design the FOSREM for registration

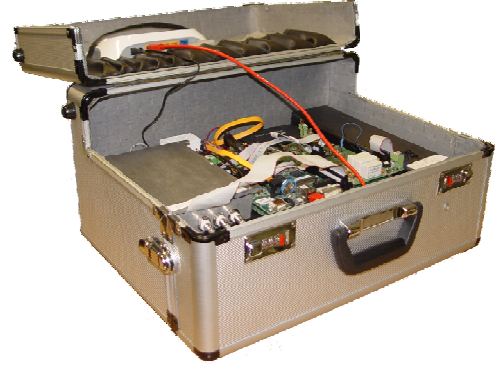

(a)

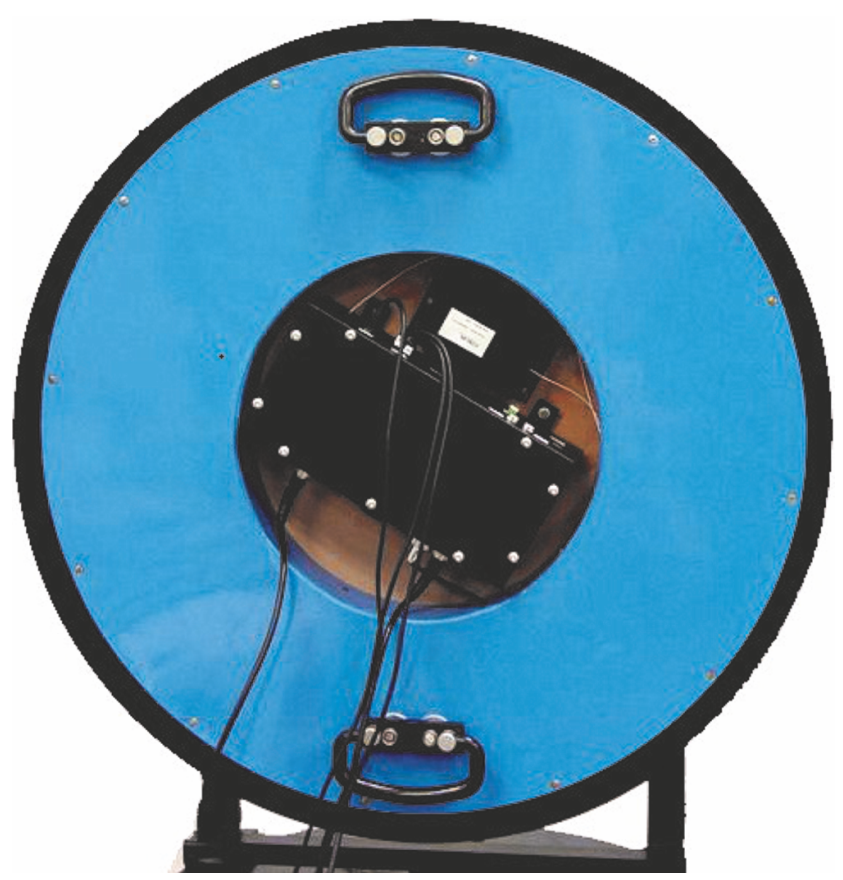

(b)

Fig. 1. Interferometric optical fibre sensors for rotation component registering: (a) FOSREM (size: $0.36 \times 0.36 \times 0.16 \mathrm{~m}$ ), (b) AFORS (size: $0.70 \mathrm{~m}$ diameter and $16 \mathrm{~cm}$ high).

of the rotation component in engineering construction, placed on the subsoil with different elastic characteristics, and allows to estimate dynamic response of these objects on various types of kinetic force during their routine operation, as well as under varying weather conditions or after earthquakes.

Physically the FOSREM bases on the widely described Sagnac effect [19] which shows the difference between phase of two beams propagating around closed optical path, in opposite direction when this path rotates with angular velocity. It allows to detect rotation in a direct way, as well as with respect to the fixed stars in the galaxy instead of the Earth's surface [21]. This attribute is very important during earthquakes when, as mentioned above, everything is unstable. The phase shift $(\Delta \phi)$ induced by the rotation component 
perpendicular to the sensor plane $(\Omega)$ does not depend on the shape of the sensor plane, as well as the centre of rotation. It is defined by the following formula [25]

$$
\Delta \phi=\frac{4 \pi R L}{\lambda c} \Omega=\frac{1}{S_{0}} \Omega,
$$

where: $L$ is the length of the fibre in a sensor loop, $R$ is the sensor loop radius, $\lambda$ is the wavelength of applied source, $c$ is the velocity of the light in vacuum, $S_{0}$ is the optical constant of interferometer.

The FOSREM construction consists of two interdependent parts: optical and electronic, described below.

\subsection{Construction of the optical part of the FOSREM}

The optical part has been performed according to, so-called, minimum gyro configuration in order to ensure the sensor's reversibility (Fig. 2).

The sensor loop has been made of a 5-km standard single-mode fibre (SMF-28) length. The SMF-28 with a catalogue attenuation that equals $0.33 \mathrm{~dB} / \mathrm{km} @ 1310 \mathrm{~nm}$ has been wound on a $0.215 \mathrm{~m}$ diameter duralumin spool. Despite of a 42 fibre layers' winding process by a double-quadrupole mode which caused bends of the optical line and introduction of microbends on the irregularities, the value of the fibre attenuation has been only slightly increased to the value of 0.35 dB/km@1310 nm (Fig. 3). The double-quadrupole mode of winding has been applied in order to exclude the influence of the thermal gradient [26] which causes inequality of optical paths of propagating beams in the sensor loop and, as a result, the introduction of an additional noise together with decreasing sensitivity of the Sagnac interferometer. This method of winding causes that two fibre lengths equidistant from the centre of the loop are placed one next to another.

The superluminescent diode (SLED) in the optical part is used as the light source with the following parameters: optical power $\mathrm{P}=11.18 \mathrm{~mW}$, central wavelength $1311.2 \mathrm{~nm}$, bandwidth of $39.7 \mathrm{~nm}$. The low coherence and high optical power of the applied SLED allow to eliminate disturbing polarization effects (polarization fading in the sensor loop $[27,28]$ ) and increasing the sensor sensitivity [27]. The fibre optic izolator with a $0.34 \mathrm{~dB}$ insertion loss and a $39 \mathrm{~dB}$ isolation is applied in order to protect the diode against backscattering. The light beam after passing through the optical fibre depolarizer is separated by an X-type coupler I. The coupler II in the optical part is added, after two fibre optic polarizes (43 $\mathrm{dB}$ extinction ratio and $0.45 \mathrm{~dB}$ insertion loss each) mounted in-line, in order to ensure the same optical path for both counter-propagating beams in the sensor loop [29]. The fibre optic polarizer assures that two both beams have the same polarization state in the first coupler. At the end of the sensor loop there is a cylindrical piezoceramic with the wo-

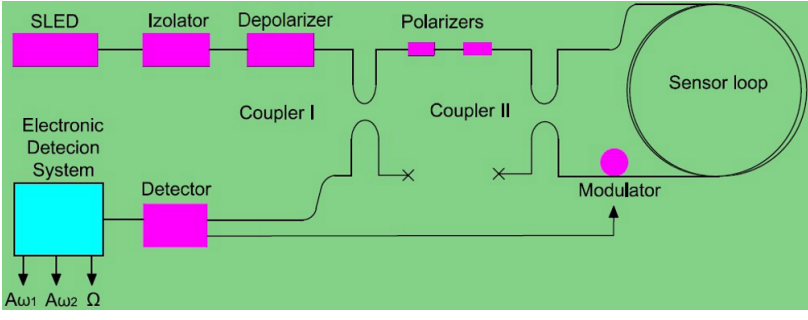

(a)

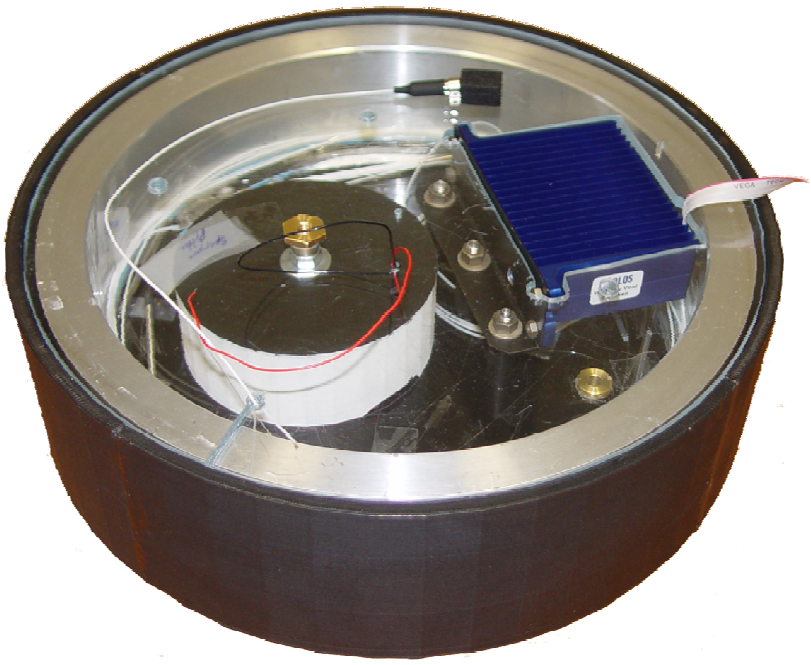

(b)

Fig. 2. Optical part of the FOSREM: (a) schematic diagram, (b) technical realization.

und single mode fibre. It performs a function of a phase modulator. The periodical deformations of the piezoceramic caused by an electrical sinusoidal signal change the length of the wound optical fibre, thereby modulating the propagating beam's phase.

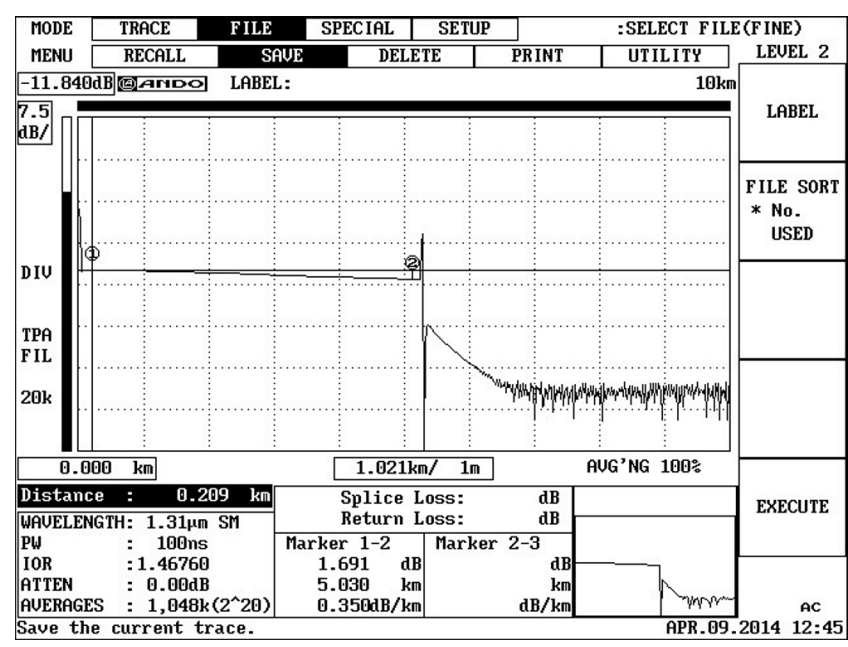

Fig. 3. The measurement of the sensor loop attenuation by OTDR AQ7250 (ANDO). 
The above described construction of the optical part ensures that the sole irreversible effect in the FOSREM is the Sagnac effect. Optical elements' parameters have been optimized for achieving the maximal detectable signal amplitude of the order of $10 \mathrm{rad} / \mathrm{s}$ and for performing a mobile system. The laboratory investigation indicated that the total attenuation of the optical part including losses introduced by particular optical components and their connections equals $16.37 \mathrm{~dB}$. Thus, theoretical sensitivity of the constructed FOSREM is equal to $2 \cdot 10^{-8} \mathrm{rad} / \mathrm{s} / \mathrm{Hz}^{1 / 2}$.

\subsection{The FOSREM detection electronic unit}

The main function of the electronic part of FOSREM is signal receiving from the detection diode, its amplification and relevant digital processing. The measurement is based on a synchronous detection for the experimentally assigned value of the frequency modulator. Frequency and amplitude of modulation have been determined taking into account the linearity of the interferometer response. The Sagnac phase shift can be determined using measurement of the first $\left(A_{1 \omega}\right)$ and second $\left(\mathrm{A}_{2 \omega}\right)$ amplitude of the harmonic output signal $[u(t)]$ by following the mathematical equation $[13,27]$

$$
\Delta \phi=\arctan \left[k \frac{A_{\omega 1}}{A_{\omega 2}}\right]=\arctan \left[\frac{u(t)}{S_{e}}\right], \quad u(t)=\frac{A_{\omega 1}}{A_{\omega 2}},
$$

where $k$ is the constant related with the phase modulator parameters, $S_{e}$ is the electronic constant of the sensor.

Then, the following formula allows for determination of the detected component of rotation [27]

$$
\Omega=S_{0} \arctan \left[\frac{u(t)}{S_{e}}\right] .
$$

In the electronic part it is necessary to apply an advanced electronic configuration due to a significant difference in the values of the first and second harmonic signal. The applied electronic solutions are currently subject of the patent procedure [30]. Generally, it is based on the appropriate filtration, amplification and digital data processing which are schematically presented in Fig. 4.

The signal processing unit calculates the rotation detected component based on Eqs. (2) and (3) by numerical processing. By taking under consideration the sensor's operation rule, FOSREM measures the rate of rotation, therefore its detection frequency bandpass has to start from $0 \mathrm{~Hz}$. The electronic part allows to measure the signal in a wide detection frequency bandpass from $0 \mathrm{~Hz}$ to the upper frequency from $2.56 \mathrm{~Hz}$ to $328.12 \mathrm{~Hz}$. The upper detection frequency bandpass determines sampling frequency and signal processing. The sampling frequency of the applied in the FOSREM's electronic part analogue-to-digital converter is equal to $672000 \mathrm{~Hz}(1,4881 \mathrm{~ms})$. In order to calculate a mea-

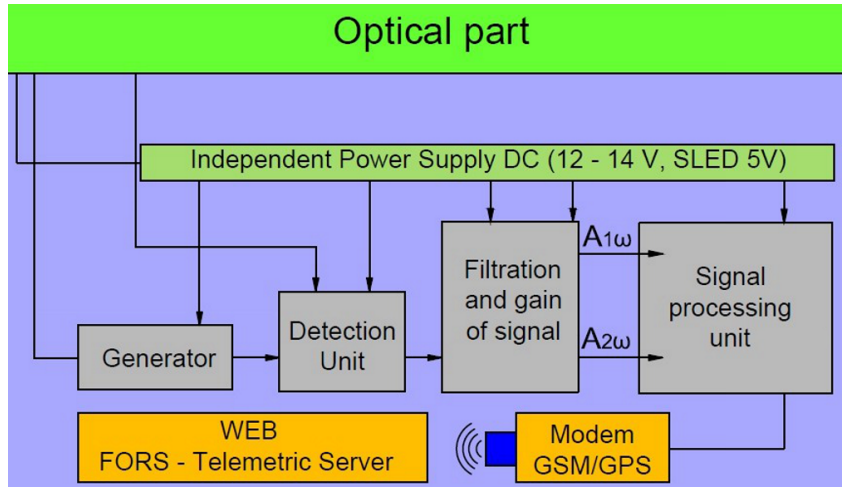

(a)

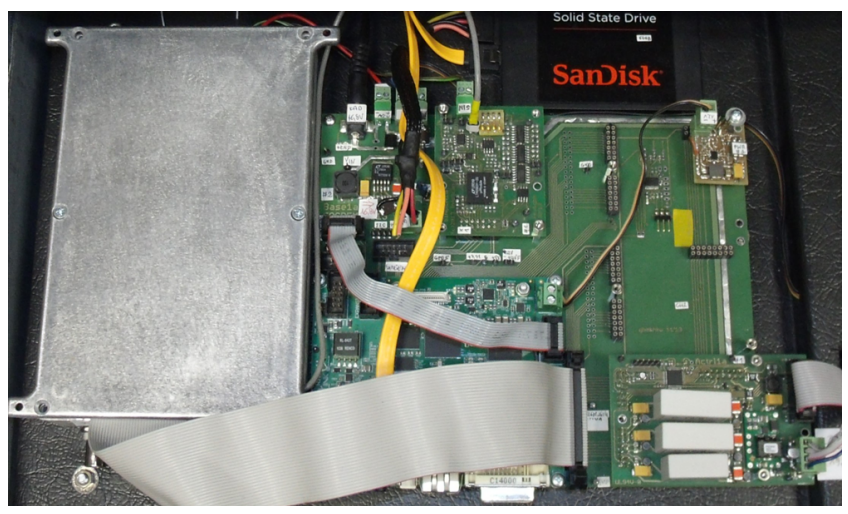

(b)

Fig. 4. The electronic part of the FOSREM: (a) block diagram, (b) components and printed circuitboards.

sured rotation rate the system receives maximum from 2 to $2^{8}=1024$ samples (in time equal to $1024 \times 1,4881 \times 10^{-6}$ $\mathrm{s}=0.00152381 \mathrm{~s}$ for 1024 samples). From the theory of sampling one needs at least two samples per period (which gives $2 \times 0,00152381 \mathrm{~s}=0,00304762 \mathrm{~s}$, and maximum detection frequency equal from $2.56 \mathrm{~Hz}$ to $1 / 0,00304762 \mathrm{~s}=328,12$ $\mathrm{Hz}$ ). In order to reduce the system's noise one applies the averaging by the basic detection time which determines how many values of the system rotation takes in calculation. This process narrows the frequency detection bandpass.

It should be emphasised that the applied measurement method in FOSREM is a completely different technical approach in comparison to Fiber Optic Gyroscope (FOG). In FOG one gains the value of the angle detected change by integration procedure of the signal calculated basing on the measurement of the Sagnac effect [19].

\subsection{The data archiving system for the FOSREM}

Additionally to electronic part functionality for detection and calculation rotational signal functions the electronic part is equipped with a high developed communication module by which the obtained data is sent to the remotely controlled 
server used for data storage. The server has an interface for communication with a local computer and a GPS/GPRS modem enabling wireless communication via network to a dedicated server. It gives an opportunity to remotely control parameters of the sensor, receiving and storage data from a distant place. The bookmarks of FOSREM software for control and receiving data are presented in Fig. 5. One can change the parameters of FOSREM's work (e.g., optical and electronic constant, amplification of the first and the second harmonic signal outputs in their canals, time constant which adjusts the sampling time, voltage value of the phase modulator) using bookmarks "System" and "Configuration". The bookmark presented in Fig. 5(b) shows rotation component value, voltage of the first and second amplitude of harmonic output signal in real time which can continue recording on server.

Despite existence of a continuous recording of the FOSREM work via internet, the method of rotational disturbance recognition with their recording is an important question. The algorithm used for the AFORS is widely described by Kurzych et al. [24] works quite correctly [see Fig. 6(a)], however, if $\mathrm{ADC}$ in the electronic part generates artefact (single data with random value over noise level) the above procedure starts recording process as is shown in Figs. 6(c)
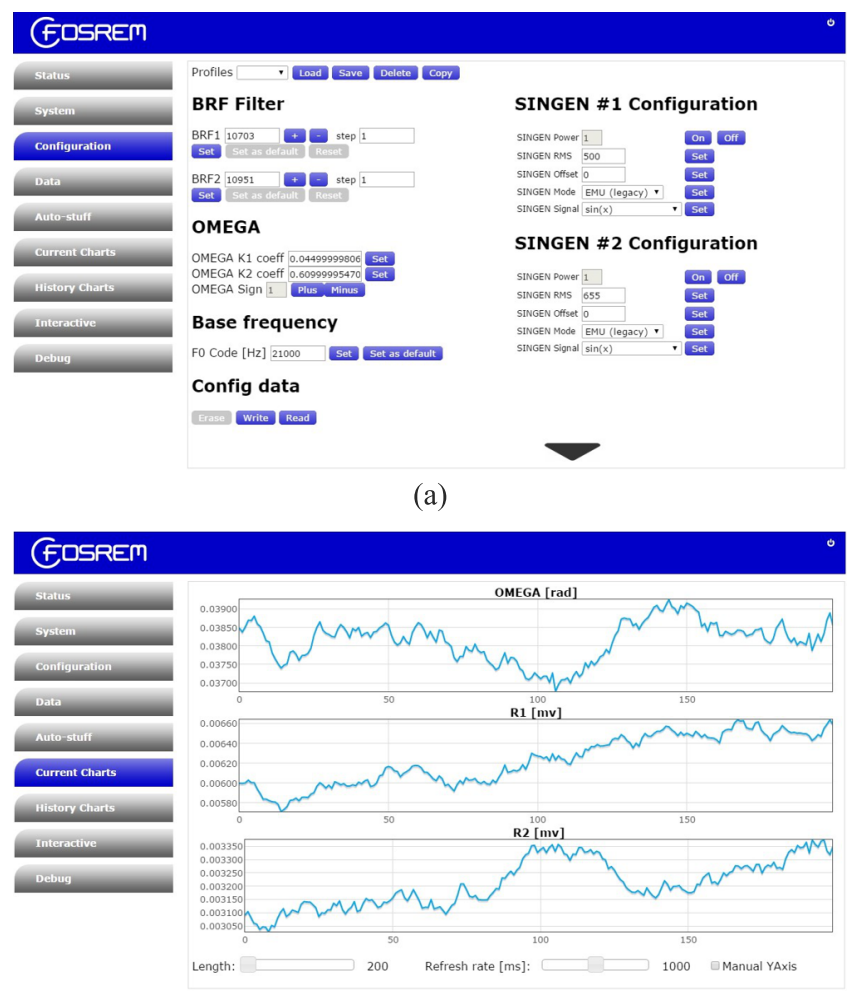

(b)

Fig. 5. The bookmarks of the FOSREM software: (a) bookmark consisting of detection settings' block, (b) panel displaying currently detected data. and 6(e). For the above reason a new algorithm, according to the following procedure, has been applied in the FOSREM. The set of $\mathrm{N}$ equal to 1024 samples of the signal is recording at the beginning and the average and standard deviations are calculated according to the formulae.

$$
\Omega_{a v}(N)=\frac{\sum_{i}^{N} \Omega_{i}}{N} \quad \Delta \Omega_{a v}(N)=\frac{\sum_{i=1}^{N}\left|\Omega_{i}-\Omega_{a v}\right|}{N} .
$$

For the next recording samples ( $>\mathrm{N}$ ), the following formulae were used for a signal average value and its standard deviation.

$$
\begin{aligned}
& \Omega_{a v}(i)=\Omega_{a v}(i-1) \frac{N-1}{N}+\Omega_{i} \frac{1}{N} \\
& \Delta \Omega_{a v}(i)=\Delta \Omega_{a v}(i-1) \frac{N-1}{N}+\left|\Omega_{a v}(i-1)-\Omega_{i}\right| \frac{1}{N} .
\end{aligned}
$$

The detection condition is established for such set of several samples with number $\mathrm{x}_{1}>\mathrm{N}(1=1,2,3)$, for which the following relation are true

$$
\begin{gathered}
\left|\Omega_{a v}\left(x_{l}\right)-\Omega_{x_{l}}\right|>\Delta \Omega_{a v}\left(x_{l}\right) k_{L} \quad l=1,2,3, \\
\Omega\left(x_{1}\right) \Omega\left(x_{2}\right)<0 ; \quad \Omega\left(x_{2}\right) \Omega\left(x_{3}\right)<0, \\
0<x_{2}-x_{1}<t_{L} ; \quad 0<x_{3}-x_{2}<t_{L},
\end{gathered}
$$

where $k_{L}$ is the chosen detection threshold and $t_{L}$ is the chosen time of detection.

The correctness of the above procedure is presented in Figs. 6(b), 6(d) and 6(f). Figure 6(b) presents the recorded data with an application of a new algorithm for data presented in Fig. 6(a). As one can see the recorded signals are identical, so it is suggested not to disturb any real rotational signal by a new algorithm. Figures 6(d) and 6(f) present the proper work of new algorithm according to artefacts, because they are obtained for data presented in Figs. 6(c) and $6(\mathrm{e})$, respectively. It was assumed that for detected by algorithm artefacts the samples with noise value are used.

\section{The results of FOSREM laboratory investigation}

In order to carry out the first experimental tests FOSREM has been mounted on the special rotational table, and standard electronic measurement device, mainly the oscilloscope and the lock-in amplifier were used (Fig. 7).

After sensor calibration by a recording rotation with a known rotation value, the calculated optical and electronic constants have been stored in the processor memory. In order to determine the accuracy of FOSREM the following methodology has been applied. The sensor has been mounted in a W-E direction which should assure the zero value 


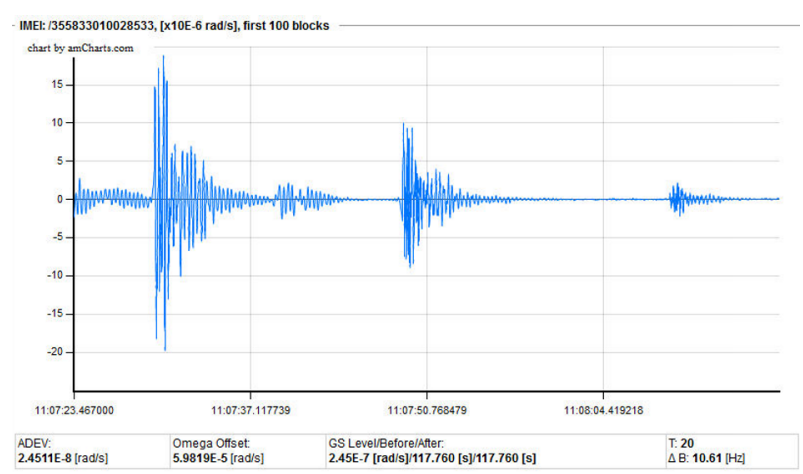

(a)

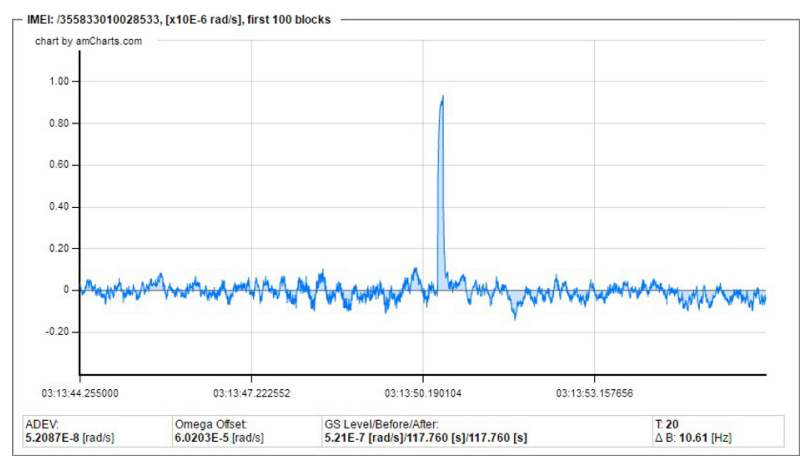

(c)

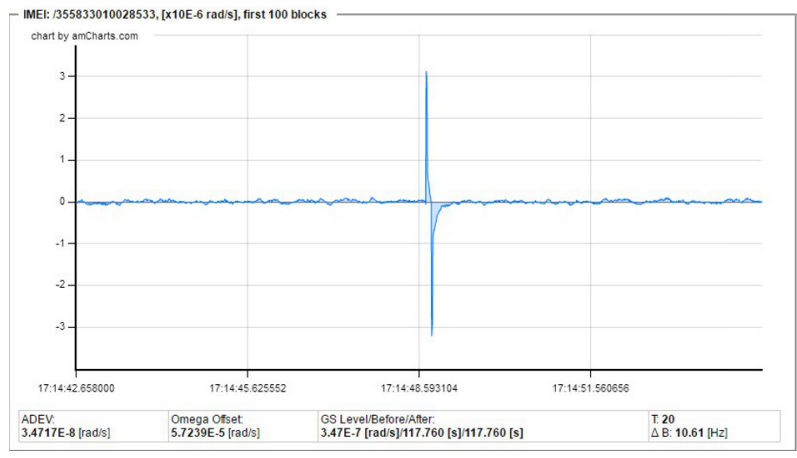

(e)

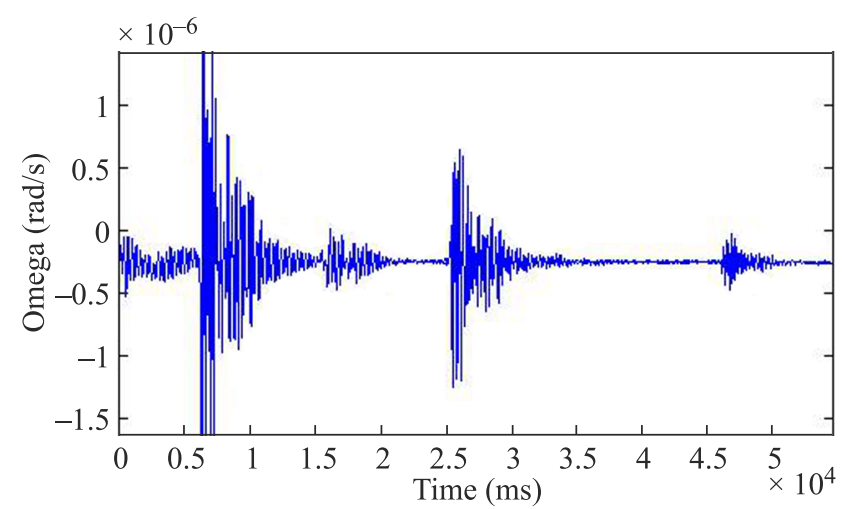

(b)

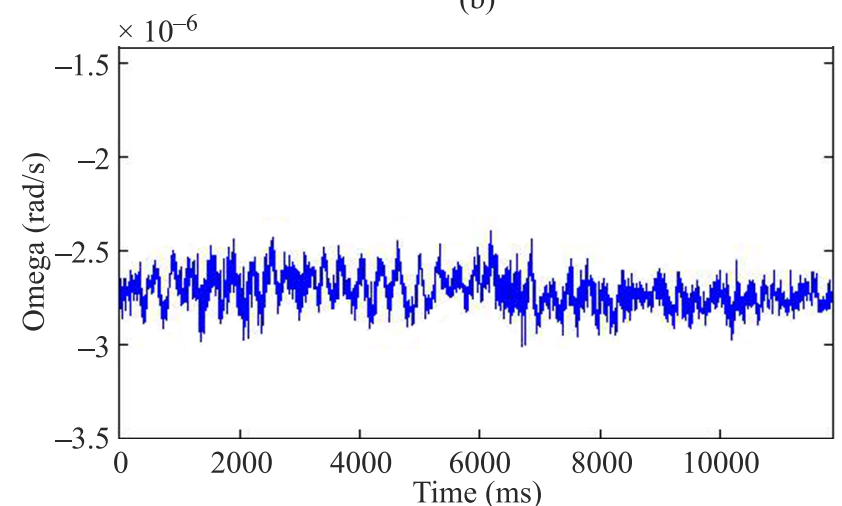

(d)

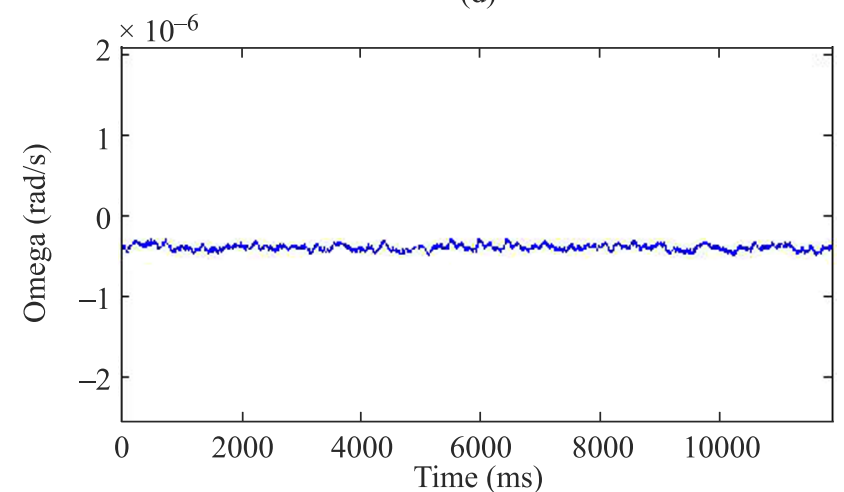

(f)

Fig. 6. Seismograms recorded by AFORS according to the old algorithm [24] as (a), (c), (e) and the same data after application of a new algorithm for FOSREM as (b), (d) and (f). For presented data the $k_{L}=10$ and $t_{L}=70$ samples were used.

of the Earth's rotation. Then, the phase modulator signal was disconnected. In such position one measures the level of noises for amplitudes $\mathrm{A}_{1 \omega}$ and $\mathrm{A}_{2 \omega}$. In order to determine the sensor's accuracy, one applied method of the errors transfer in indirect measurements. The rotation is calculated basing on the measurement of two values: the first $\left(\mathrm{A}_{1 \omega}\right)$ and the second $\left(\mathrm{A}_{2 \omega}\right)$ amplitude of harmonic output signal by the following equation [21]

$$
\Omega=S_{0} \arctan \left[S_{e} \frac{A_{\omega 1}}{A_{\omega 2}}\right] .
$$

The accuracy of rotation rate measured by FOSREM for a given measurement frequency bandpass can be found by a standard error equation application which gives the following dependence [21]

$$
\Delta \Omega=\sqrt{\left(\frac{\partial \Omega}{\partial A_{1 \omega}} \sigma A_{1 \omega}\right)^{2}+\left(\frac{\partial \Omega}{\partial A_{2 \omega}} \sigma A_{2 \omega}\right)^{2}},
$$

where: $\sigma A_{1 \omega}, \sigma A_{2 \omega}$ are the mean values of noises recorded for the amplitudes $A_{1 \omega}$ and $A_{2 \omega}$, respectively. The exemplary measurement of noises of the signal components $A_{1 \omega}$ and $A_{2 \omega}$ in 0-328.12 Hz detection bandpass are presented in Fig. 8. 


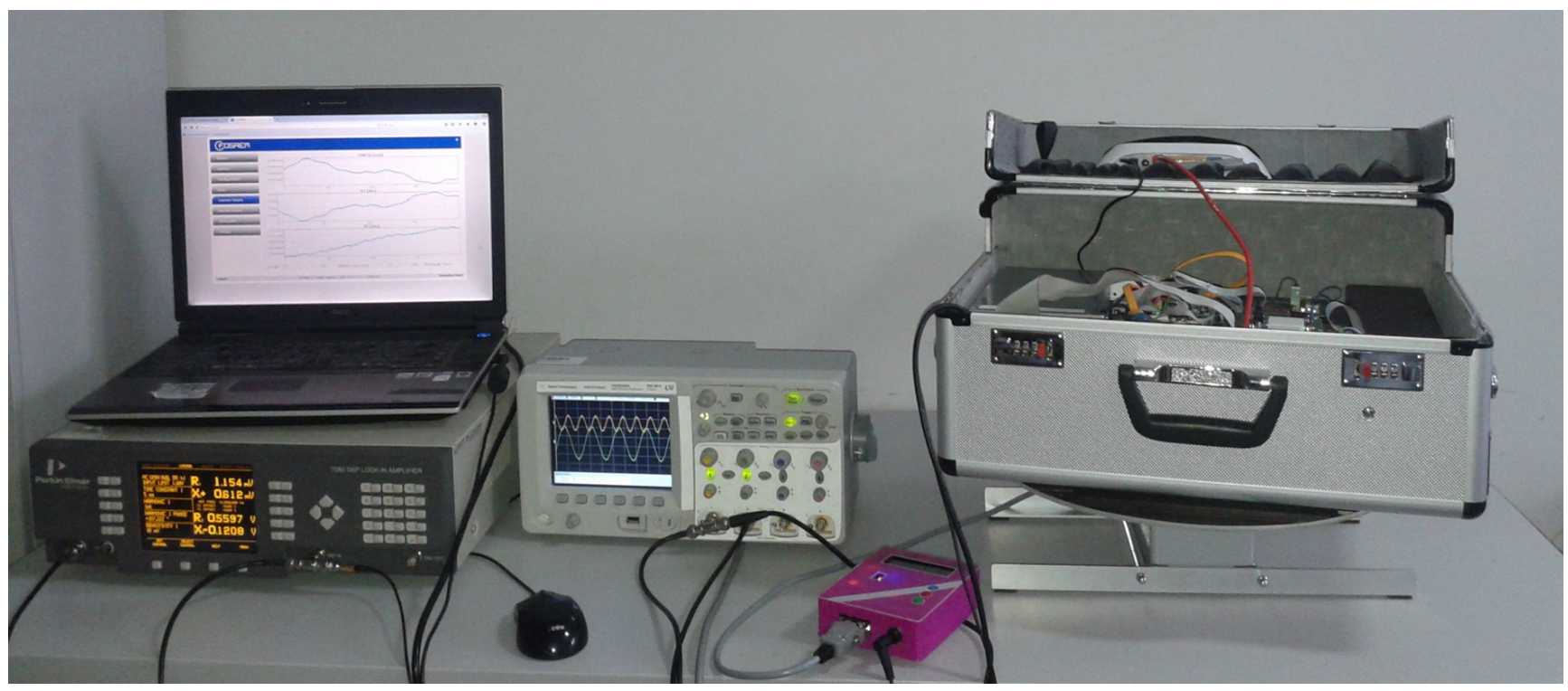

Fig. 7. The measuring position for FOSREM calibration and testing.

As has been mentioned above, the electronic part of FOSREM allows for step changes of the detection frequency bandpass in the range from $0 \mathrm{~Hz}$ to the upper frequency between $2.56 \mathrm{~Hz}$ and $328.12 \mathrm{~Hz}$, the above procedure gives accuracy from $3 \cdot 10^{-8}$ to $1.6 \cdot 10^{-6} \mathrm{rad} / \mathrm{s}$ which is presented in Fig. 9.

The first measurements of the Earth's rotation component in Warsaw (equal to $4.45 \cdot 10^{-5} \mathrm{rad} / \mathrm{s}$ ) for different value of detection frequencies' bandpass were carried out after FOSREM calibration. The obtained data are presented in Fig. 10.

The presented graphs confirm the opportunity to detect rotational movements by FOSREM in a wide range of frequency which is necessary for monitoring torsional effects in the engineering constructions. Moreover, the special constructed rotational table enables to carry out FOSREM measurements in a wide range of signal amplitude. The measurements at a signal amplitude range of $25 \mathrm{mrad} / \mathrm{s}-1 \mathrm{rad} / \mathrm{s}$ have been carried out using the measuring position presented in Fig. 7. The obtained data are presented in Fig. 11 that confirms the FOSREM proper operation in a whole amplitude range of the rotational movement.

Finally, the FOSREM thermal stability was controlled due to the FOSREM application in the engineering construction where there can be various thermal conditions. The measurements were carried out at a temperature range of $0^{\circ} \mathrm{C}-50^{\circ} \mathrm{C}$ in a climatic chamber VCL 7010 (Fig. 12). The temperature change was controlled automatically by a PLC programmer in a continuous way. After each temperature jump equals $10^{\circ} \mathrm{C}$ the programmer kept temperature at constant level in order to stabilize thermal condition in the entire volume of the chamber and FOSREM. The obtained data

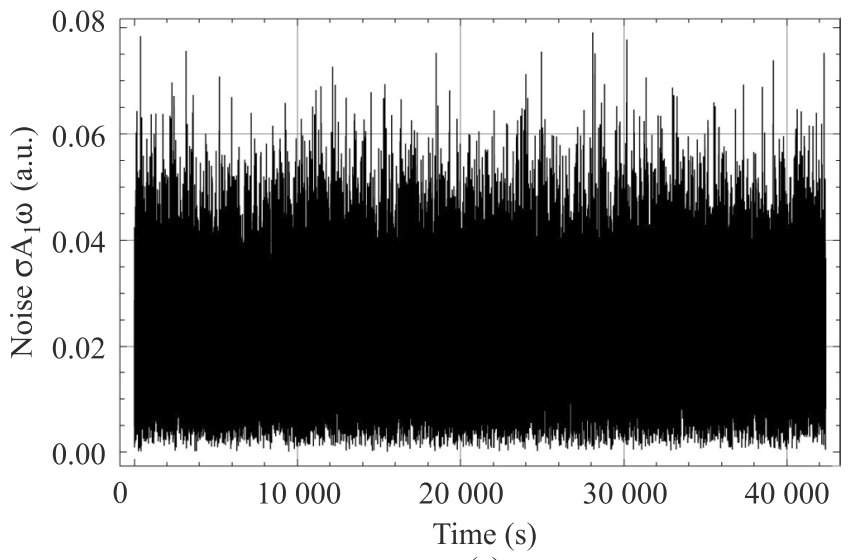

(a)

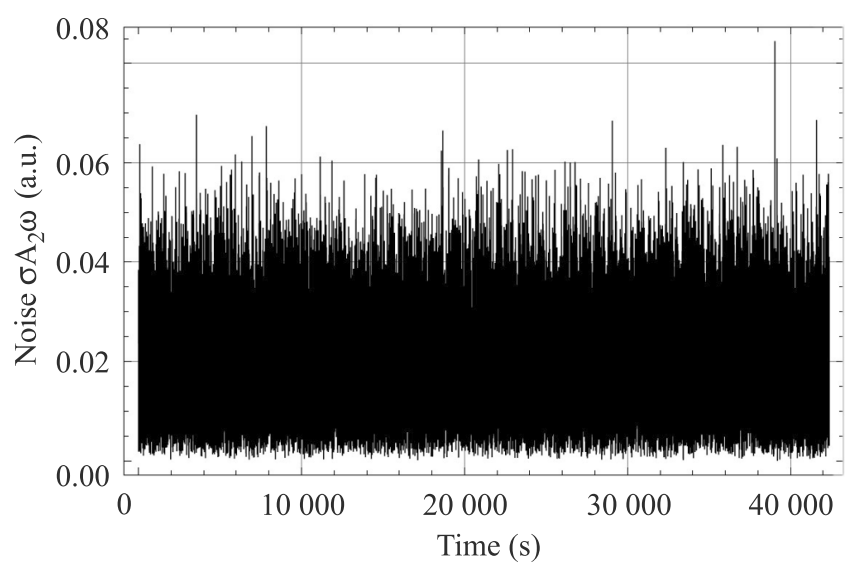

(b)

Fig. 8. The noise in 0-328.12 Hz detection frequency bandpass for the first $A_{1 \omega}$ (a) and the second $A_{2 \omega}$ (b) signal components. 


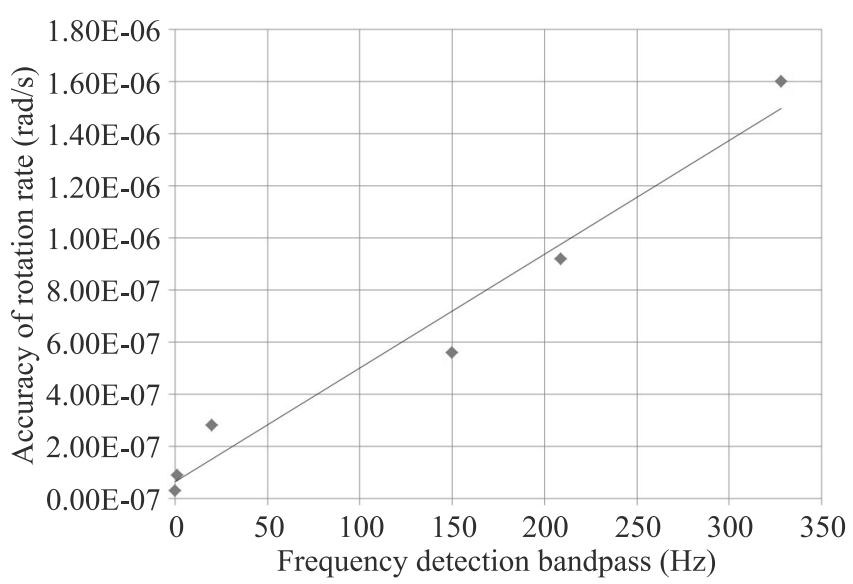

Fig. 9. The FOSREM accuracy measured for the chosen detection frequency bandpass.

presented in Fig. 13, as well as calculated for them signal instabilities (Table 1) show that FOSREM is stable at a temperature range of $0^{\circ} \mathrm{C}-50^{\circ} \mathrm{C}$. The signal jumps are negligible and are result from vibrations introduced by the climatic chamber work which generates forced air cycle.

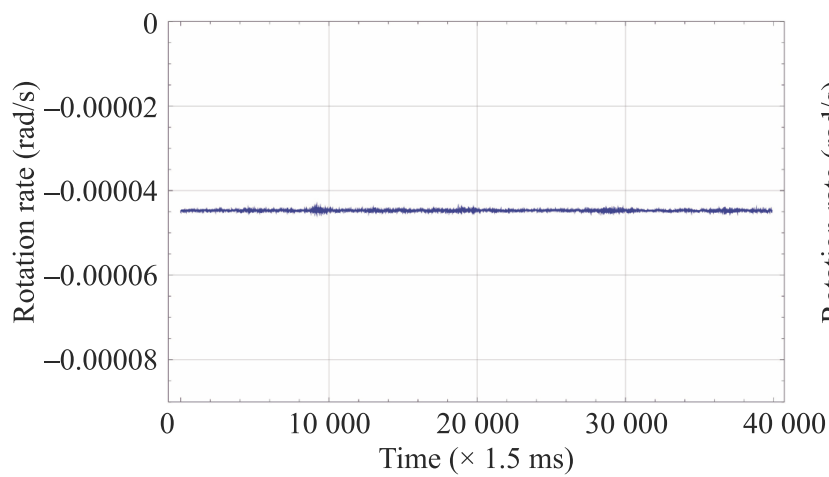

(a)

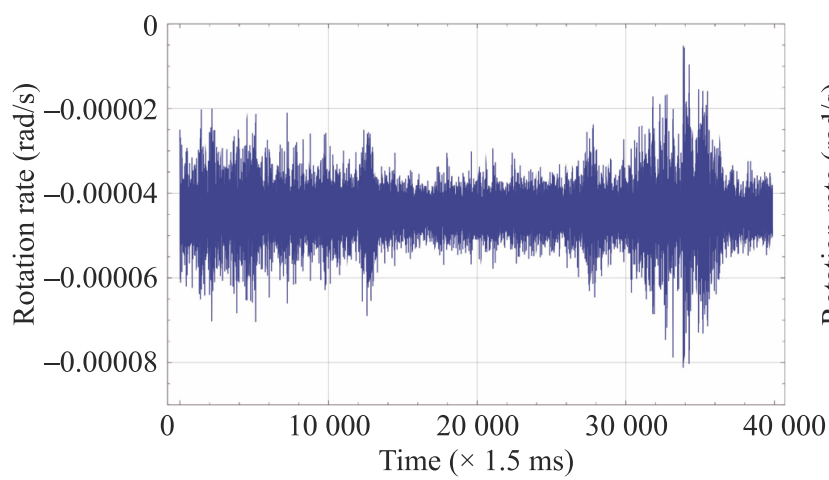

(c)

\section{Conclusions}

In this work the construction and performance of the innovative optical fibre sensor - FOSREM have been presented. FOSREM's laboratory investigations confirm its usefulness for continuously monitoring of rotational components, both in civil engineering constructions and seismological observatories. For complex and large-size constructions, where their asymmetries can cause torsional effects and inter-storey drifts, FOSREM guarantees a wide range of the detected signal amplitude from $10^{-8} \mathrm{rad} / \mathrm{s}$ to $10 \mathrm{rad} / \mathrm{s}$, as well as a wide range of the frequency from 0 to the upper frequency from $2.56 \mathrm{~Hz}$ to $328.12 \mathrm{~Hz}$ which is very significant during measurements in the civil engineering constructions.

One of the main advantages of the FOSREM is its equipment in the advanced communication module which gives a possibility of remotely detection parameter control, as well as the recorded data receiving. It enables to assemble the senor in any chosen place.

It should be emphasised that FOSREM has a wide application field including the classic research in seismological observatories, as well as geological measurements of unidirectional rotations connected with landslides, for example in mines, glaciers, etc. Authors believe that data recorded in the near term by FOSREM will significantly contribute to extend the knowledge about torsional vibrations and inter-

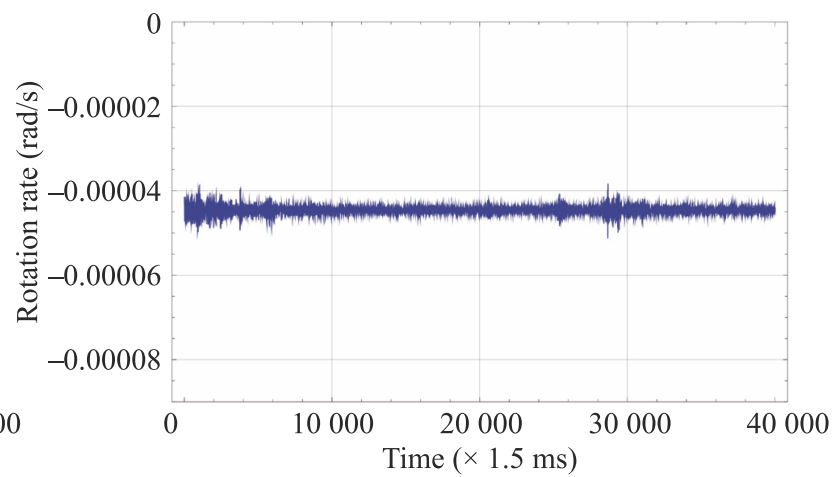

(b)

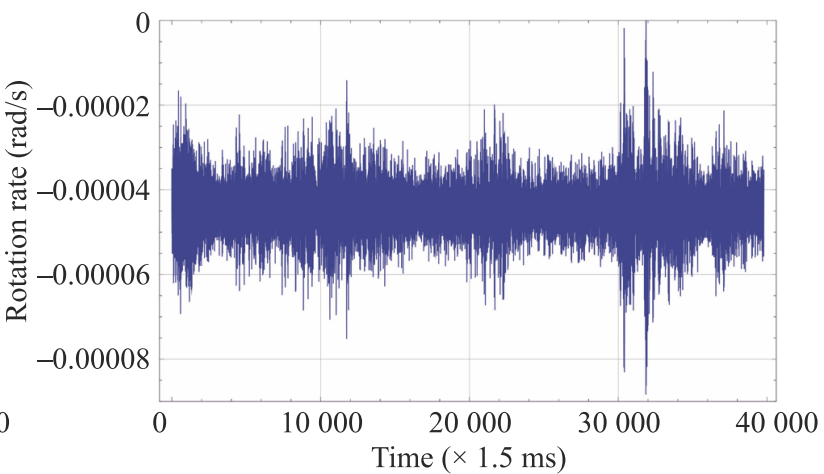

(d)

Fig. 10. Example of measured rotation rate at a various frequency bandpass: (a) $2.56 \mathrm{~Hz}$; (b) $54.69 \mathrm{~Hz}$; (c) $109.38 \mathrm{~Hz}$; and (d) $328.12 \mathrm{~Hz}$. 


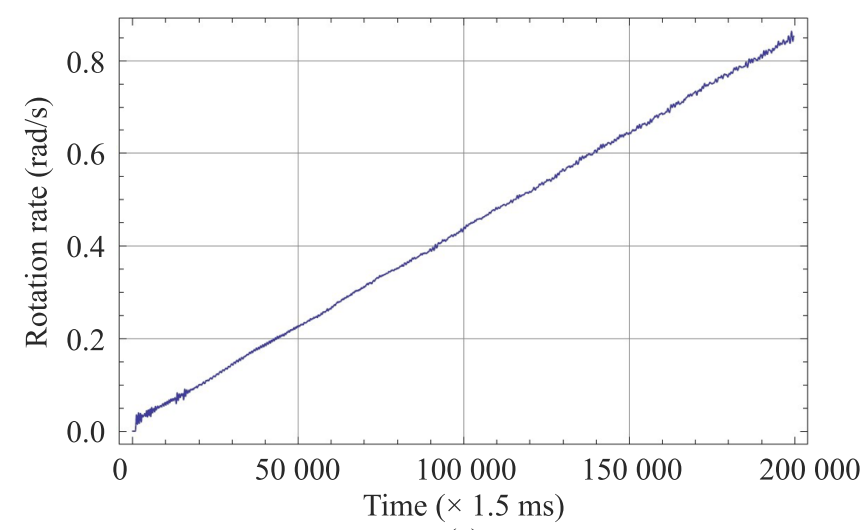

(a)

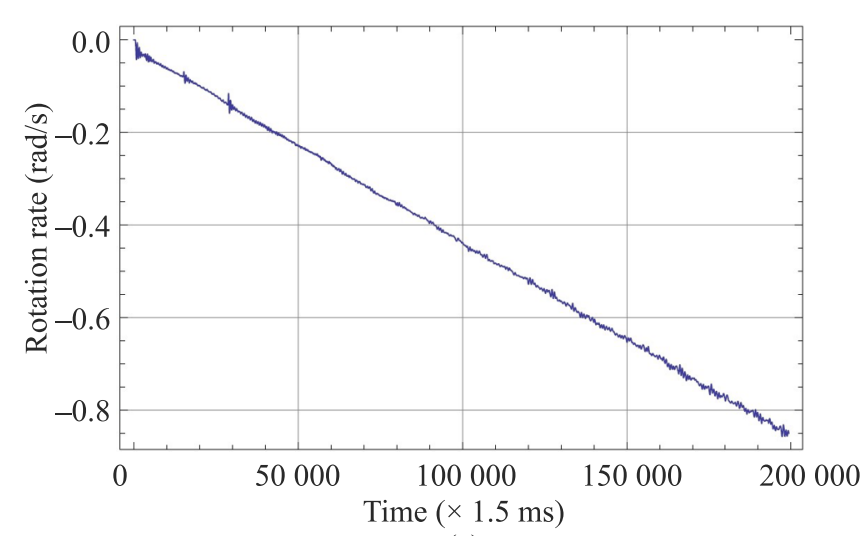

(a)

Fig. 11. Example of measured rotation rate at a signal amplitude range $\pm(25 \mathrm{mrad} / \mathrm{s}-1 \mathrm{rad} / \mathrm{s})$ carried out on the special designed rotational table.

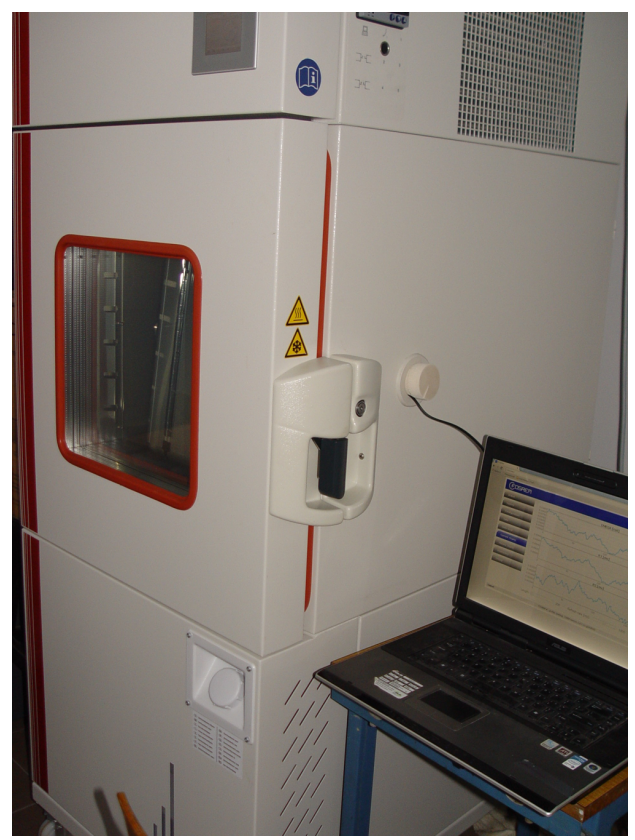

Fig.12. Climatic chamber VCL 7010P applied to thermal measurements.

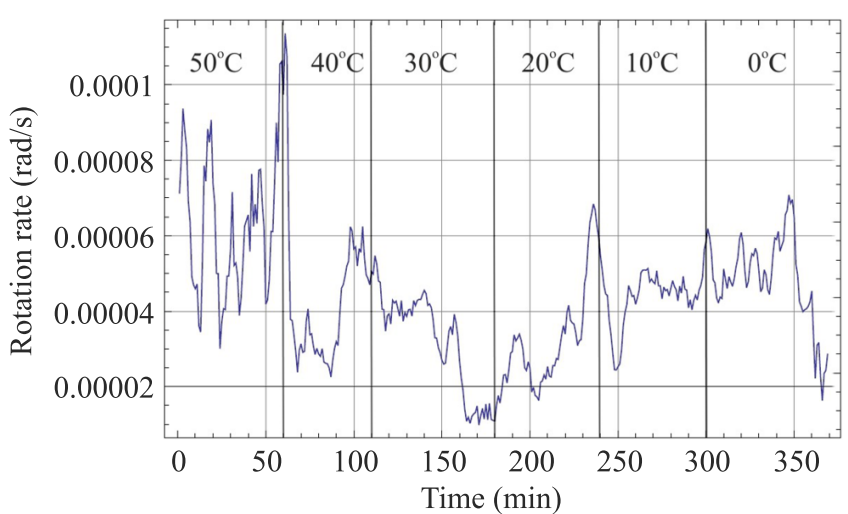

(a)

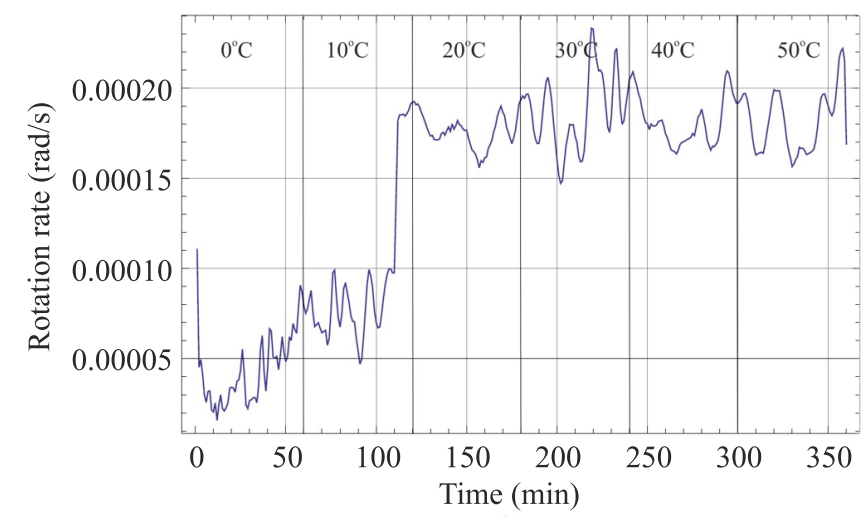

(b)

Fig. 13. The measurements in the climatic chamber at a temperature range of $0^{\circ} \mathrm{C}-50^{\circ} \mathrm{C}$ : (a) cooling process, and (b) heating process.

-storey drifts in the complex engineering constructions, as well as rotational events associated with an earthquake.

\section{Acknowledgements}

This work was done under the financial support of the Polish Ministry of Science and Higher Education under contract No PBS1/B3/7/2012 and statutory activity of Military University of Technology PBS-654.

\section{References}

1. H. Igel, J. Brokesova, J. Evans, and Z. Zembaty, "Preface to the special issue on advances in rotational seismology: instrumentation, theory, observations and engineering", J. Seismol. 16, 571-572 (2012).

2. W.H.K. Leee, M. Celebi, M.I. Todorovska, and H. Igel, "Introduction to the special issue on rotational seismology and enginering applications", Bull. Sesimol. Soc. Am. 99, 945-957 (2009).

3. A.C. Eringen, Mirocontinuum field theories. Vol. 1 Foundations and Solids, Springer-Verlag, ISNB 0-387-95275-6, New York, 1999. 
Table 1. Maximum signal instability $\sigma$.

\begin{tabular}{lcccccc}
\hline Temp. & $0^{\circ} \mathrm{C}$ & $10^{\circ} \mathrm{C}$ & $20^{\circ} \mathrm{C}$ & $30^{\circ} \mathrm{C}$ & $40^{\circ} \mathrm{C}$ & $50^{\circ} \mathrm{C}$ \\
\hline Cooling $[\sigma]$ & $4.42 \cdot 10^{-4}$ & $1.27 \cdot 10^{-4}$ & $1.63 \cdot 10^{-4}$ & $0.98 \cdot 10^{-4}$ & $6.42 \cdot 10^{-4}$ & $5.86 \cdot 10^{-4}$ \\
Heating $[\sigma]$ & $3.50 \cdot 10^{-4}$ & $1.35 \cdot 10^{-3}$ & $1.16 \cdot 10^{-4}$ & $1.46 \cdot 10^{-4}$ & $1.42 \cdot 10^{-4}$ & $1.67 \cdot 10^{-4}$ \\
\hline
\end{tabular}

4. R. Teisseyre and W. Boratyński, "Continuum with self-rotation fields: evolution of defect fields and equations of motion”, Acta Geophys. 50, 223-229 (2002).

5. R. Teisseyre, M. Białecki, and M. Górski, "Degenerated mechanics in a homogeneous continuum: potentials for spin and twist", Acta Geophys. 53, 219-223 (2005).

6. R. Teisseyre and M. Gorski, "Transport in fracture processes: fragmentation of defect fields and equations of motion", Acta Geophys. 57, 583-599 (2009).

7. Z. Droste and R. Teisseyre, "Rotational and displacemental components of ground motion as deduced from data of the azimuth system of seismograph", Publs Inst. Geophys. Pol. Acad. Sc. 97, 157-167 (1976).

8. T. Moriya and R. Teisseyre, "Discussion on the recording of seismic rotation waves", Acta Geophys. Pol. 47, 351-362 (1999).

9. U. Schreiber, M. Schneider, C.H. Rowe, G.E. Stedman, and W. Schlüter, "Aspects of ring lasers as local earth rotation sensors", Surveys in Geoph. 22, 603-611 (2001).

10. L.R. Jaroszewicz, Z. Krajewski, L. Solarz, P. Marć, and T. Kostrzyński, "A new area of the fiber-optic Sagnac interferometer application", Intern. Microwave and Optoelectronics Conference, Iguazu Falls, 661-666, 2003.

11. L.R. Jaroszewicz, Z. Krajewski, L. Solarz, and R. Teisseyre, "Application of the fibre-optic Sagnac interferometer in the investigation of seismic rotational waves", Meas. Sci. Technol. 17, 1186-1193 (2006).

12. U. Schreiber, G.E. Stedman, H. Igel, and A. Flaws, "Ring laser gyroscopes as rotation sensors for seismic wave studies", in: R. Teisseyre, M. Takeo, E. Majewski, (Eds) Earthquake Source Asymmetry, Structural Media and Rotation Effects, Springer-Verlag Berlin Heidelberg, 29, 377-390, (2006).

13. Z. Zembaty, A. Rossi, and A. Spagnoli, "Estimation of rotational groung motion effects on the bell Tower of Parma cathedral", in: Zembaty Z., and De Stefano M. (Eds) Seismic Behaviour and Design of Irregular and Complex Civil Structures II, Springer, Dordrecht, Part I.4, 35-48, 2016.

14. K. U. Schreiber, A. Velikoseltsev, A. J. Carr, and R. Franco-Anaya, "The application of fiber optic gyroscopes for the measurement of rotations in structural engineering", Bull. Sesimol. Soc. Am. 99, 1207-1214 (2009).

15. Z. Zembaty, S. Kokot, and P. Bobra, "Application of rotational rate sensors in measuring beam flexure and structural health monitoring" in: Zembaty Z., and De Stefano M. (Eds) Seismic Behaviour and Design of Irregular and Complex Civil Structures II, Springer, Dordrecht, 6, 65-76, 2016.

16. R. Franco-Anaya, A.J. Carr, and K U. Schreiber, "Laboratory and in-situ measurements of structural rotations using fibre-optic gyroscopes", The Fifthteenth World Conference On Earthquake Engineering, Lisbon, 2012.
17. McGinnis "Apparatus and method for detecting deflection of a tower", U.S. Patent application, No.0107671 A1, 2004.

18. V. Gicev and M.D. Trifunac, "2009 Rotations in a shear-beam model of a seven-story building caused by nonlinear waves during earthquake excitation", Struct. Control Hlth. 16, 460-82 (2009).

19. G. Sagnac, "The light ether demonstrated by the effect of the relative wind in ether into a uniform rotation interferometer", Acad. Sci. 95, 708-710 (1913).

20. Z. Zembaty, S. Kokot, and P. Bobra, "Application of rotation rate sensors in an experiment of stiffness "reconstruction", Smart Mater. Struct. 22, 077001 (2013).

21. L. Jaroszewicz, Z. Krajewski, H. Kowalski, G. Mazur, P. Zinówko, and J. Kowalski, "AFORS autonomous fibre-optic rotational seismograph: Design and application”, Acta Geophys. 59, 578-596 (2011).

22. L.R. Jaroszewicz, Z. Krajewski, and K.P. Teisseyre, "Usefulness of AFORS - Autonomous Fibre-Optic Rotational Seismograph for investigation of rotational phenomena", J. Seismol. 16, 573-586 (2012).

23. A. Kurzych, K. P. Teisseyre, Z. Krajewski, and L. R. Jaroszewicz, "Rotational Components of the Seismic Fields Caused by Local Events", in: Abbas M. (Ed) Earthquake engineering - form engineering seismology to optimal seismic design of engineering structures, Intech Rijeka, Croatia, 6, 163-188, 2015.

24. A. Kurzych, L.R. Jaroszewicz, Z. Krajewski, K.P. Teisseyre, and J.K. Kowalski, "Fibre optic system for monitoring rotational seismic phenomena", Sensors 14, 5459-5469 (2014).

25. E.J. Post, "Sagnac effect", Rev. Mod. Phys. 39, 475-493 (1967).

26. X. Dai, X. Zhao, B. Cai, G. Yang, K. Zhou, and C. Liu, "Quantitative analysis of the Shupe reduction in a fiber-optic Sagnac interferometer", Opt. Eng. 41, 1155-1156 (2002).

27. Z. Krajewski, "Fiber-optic Sagnac interferometer as System for rotational phenomena investigation connected with seismic events", [IN POLISH]. Doctoral Thesis, MUT, Poland 2005.

28. Z. Krajewski, L.R. Jaroszewicz, and L. Solarz, "Optimization of fiber-Optic Sagnac interferometer for detection of rotational seismic events", Proc. of SPIE 5952, 240-248 (2005).

29. E. Udd and B.W. Spillman, Fiber Optic Sensors, New Jersey: John Wiley\&Sons, Inc., 2011.

30. J.K. Kowalski, L.R. Jaroszewicz, Z. Krajewski, A. Kurzych, and P. Marć, "Measurement method and system for measuring amplitude of first two harmonics of signal derived from Sagnac system", patent application PCT/IB2015/059521 from 10-12-2015. 